\title{
European association for clinical pharmacology and therapeutics young clinical pharmacologists working group: a cornerstone for the brighter future of clinical pharmacology
}

\author{
Andrej Belančić ${ }^{1}\left[\right.$ - Carla Sans-Pola ${ }^{2} \cdot$ Emilie Jouanjus $^{3} \cdot$ Pau Alcubilla ${ }^{4} \cdot$ Ana Lucía Arellano $^{4} \cdot$ Miodrag Žunić $^{5}$. \\ Rita Nogueiras-Álvarez $z^{6} \cdot$ Rossana Roncato ${ }^{7,8} \cdot$ Joaquín Sáez-Peñataro ${ }^{4}$ on behalf of the EACPT Young Clinical \\ Pharmacologists Working Group
}

Received: 7 December 2021 / Accepted: 10 January 2022 / Published online: 17 January 2022

(c) The Author(s), under exclusive licence to Springer-Verlag GmbH Germany, part of Springer Nature 2022

\begin{abstract}
The European Association for Clinical Pharmacology and Therapeutics (EACPT) is a leading society in Europe serving the European and global Clinical Pharmacology and Therapeutics community. Its specific aims include promotion of the utilisation and divulgation of the utility of clinical pharmacology services in health care delivery. EACPT currently has four active working groups (WGs): Education, Regulatory affairs, Clinical research and Young Clinical Pharmacologists (YCP WG). EACPT YCP WG was established in 2015 with the idea of improving education, research, training and networking/ mobility opportunities for YCPs across Europe and globe. The main objective of the present manuscript is to provide detailed information on general characteristics, structure, chronogram, objectives, accomplishments and current/future focus areas of the EACPT YCP WG. Consequently, we tend to notably enhance EACPT YCP WG's visibility, increase the number of its members and mobility/networking options and to expand areas of activity even more. Moreover, by this we can also make clinical pharmacology more attractive to early career fellows and colleagues and empower its position alongside other medical specialties.
\end{abstract}

Keywords Clinical pharmacology $\cdot$ Education $\cdot$ Medical specialty $\cdot$ Mobility $\cdot$ Research $\cdot$ Social networking

Andrej Belančić

a.belancic93@gmail.com

1 Department of Clinical Pharmacology, Clinical Hospital Centre Rijeka, Krešimirova 42, 51000 Rijeka, Croatia

2 Department of Clinical Pharmacology, Vall d'Hebron Hospital Universitari, Barcelona, Spain

3 Addictovigilance Center, Department of Medical Pharmacology, Toulouse University Hospital, Toulouse, France

4 Department of Clinical Pharmacology, Hospital Clinic of Barcelona, Barcelona, Spain

5 Department of Anesthesiology, Intensive Care and Pain Management, University Medical Center Maribor, Maribor, Slovenia

6 Department of Clinical Pharmacology, Badajoz University Hospital, Badajoz, Extremadura, Spain

7 Experimental and Clinical Pharmacology Unit, Centro Di Riferimento Oncologico Di Aviano (CRO) IRCCS, Aviano, Italy

8 Italian Society of Pharmacology (SIF), Milan, Italy

\section{Introduction}

Clinical pharmacology (CP) is a scientific discipline and medical speciality that involves all aspects of the relationship between drugs and humans. Thus, it has a complex and broad scope, from drug discovery and development, to study of biomarkers, pharmacokinetics, drug metabolism, regulatory issue and pharmacovigilance [1-4]. Clinical pharmacologists work in academia, industry, hospital and/or government to personalize treatment based on pharmacogenetics and pharmacokinetics parameters, investigate adverse reactions and interactions, evaluate and design clinical trials, develop clinical guidelines for rational drug use, among other activities. Young clinical pharmacologists (YCPs) take an active role in all these areas since the early beginnings of their training period. The European Association for Clinical Pharmacology and Therapeutics (EACPT), which is a leading society in Europe serving the European and global Clinical Pharmacology and Therapeutics community, has specific aims including promotion of the utilisation and 
divulgating the utility of $\mathrm{CP}$ services in health care delivery. The EACPT currently has four active working groups (WGs): Education, Regulatory affairs, Clinical research and Young Clinical Pharmacologists WG [5-7]. The main objective of the present manuscript is to provide information on general characteristics, organization and focus areas of the EACPT YCP WG. The latter WG was established in 2015 with the idea of improving education, research, training, and networking/mobility opportunities for YCPs across Europe and globe $[5,8,9]$. Number of active WG members has been gradually rising since then. Thus, besides our two co-chairs (Emilie Jouanjus from France and Carla SansPola from Spain), we currently have 23 active members from across Europe (Spain $n=10$; Lithuania $n=2$; Russia $n=2$; Sweden $n=2$; Croatia $n=1$; Cyprus $n=1$; Italy $n=1$; Norway $n=1$; Portugal $n=1$; Slovenia $n=1$; United Kingdom $n=1$ ) and are constantly recruiting/welcoming new motivated YCPs.

\section{Primary goals of the working group}

Since its beginning in 2015, the EACPT YCP WG has been focusing efforts to become a networking platform that would enable YCPs to seek out and establish collaborative projects at the European level. The WG should also be seen as a unique opportunity to consolidate networking and strengthen collaborative bonds between trainees, junior and senior clinical pharmacologists. It enables, in a direct and straightforward manner, the possibility of contacting other $\mathrm{CP}$ colleagues through regular meetings to propose and discuss collaborative research ideas.
Specifically, the YCP pre-meetings, organized within the framework of the biannual EACPT Congresses, are a great scenario to establish new collaborations and research project ideas. This space was conceived as a forum, to discuss important issues regarding $\mathrm{CP}$ as training programs and their evident differences across Europe (Fig. 1).

The WG is represented in the EACPT Executive Committee through co-opted members who report the group activities in a timely manner. The co-opted members to the EACPT executive committee representing the WG are the current chairs of the group. This representation enables the possibility to propose to the EACPT the establishment of collaborative research and mobility grants aimed to YCPs in Europe.

Thus, the overall philosophy and the "raison d'être" of this WG could be summarized in three general axes of action, namely: (1) creation of a regular networking framework for YCPs from all over Europe to enable international collaborations. The nature of such collaborations could be either scientific (in the form of joint research projects) or educational (in the form of establishing training programs between candidates and host institutions). In this regard, the organisation of the Congress pre-meetings and the group periodic meetings are crucial; (2) serve as a forum to unify all European trainees/YCPs under a common umbrella to address future challenges in an aligned manner of our medical specialty; (3) interact with the EACPT and other CP WGs, as well as with other societies of other specialties to pursue the above-mentioned objectives and divulgate the benefits of implementing clinical pharmacologist figure in healthcare and increase the visibility of CP and of YCPs.

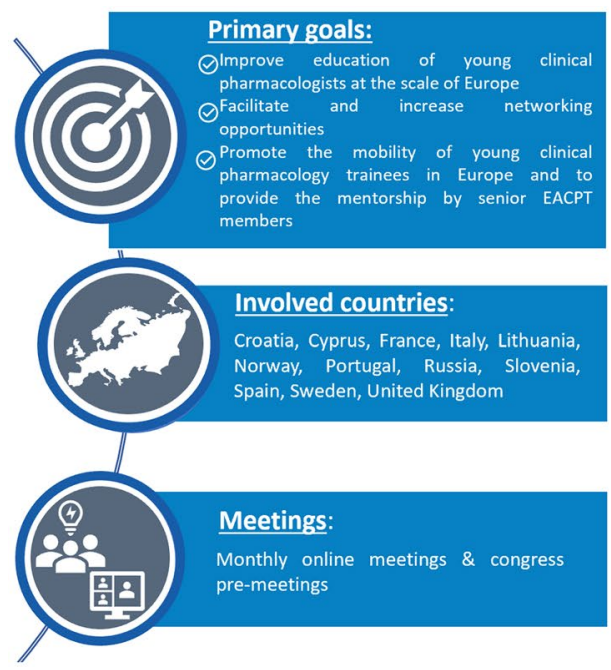

\section{TIMELINE of EACPT \\ Young Clinical Pharmacologists - Working Group}

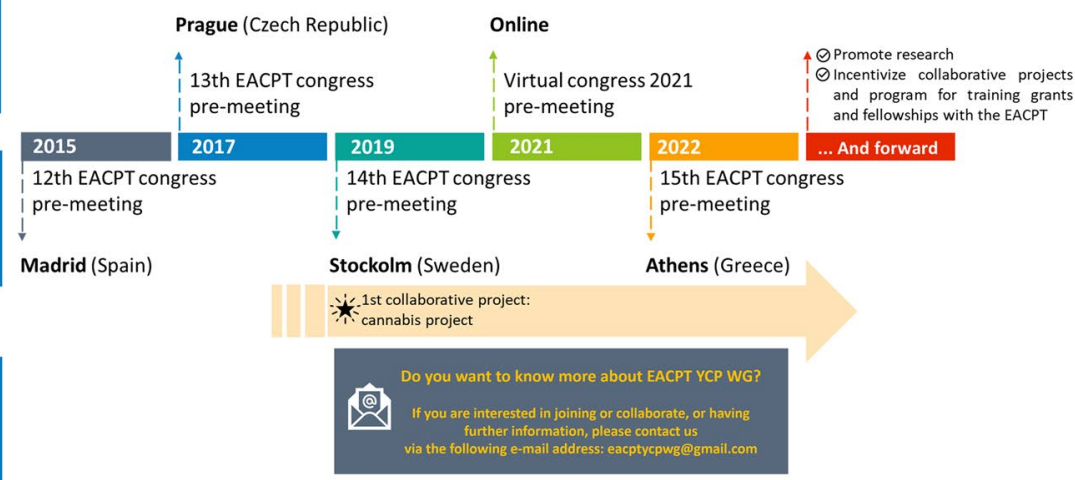

Fig. 1 The main characteristics of the European Association for Clinical Pharmacology and Therapeutics Young Clinical Pharmacologists Working Group (EACPT YCP WG) 


\section{Beginnings of the working group. From idea inception to foundational meeting (2014- 2017)}

A European Task Force with Clinical Pharmacology representatives was created in 2014 to identify main priorities in CP education across Europe. An online survey was created to explore urgent needs in terms of education and career opportunities. The results were discussed in a meeting held before the 2015 European Congress, where young and senior researchers in $\mathrm{CP}$ from different countries were invited to attend [8]. As the main output, the creation of an international Network of young researchers was proposed. As mentioned before, the Network was materialized in a WG with three primary objectives: education, mobility and networking. The first commitment of the WG was to make young researchers more visible. Therefore, a section dedicated to the YCP WG was added on the webpage of the EACPT Society. Additionally, a representative of the WG was elected as co-opted member of the Executive Committee, and it was agreed to honour a young investigators' pre-meeting before each Congress. Among others, those pre-meetings were also seen as great opportunities for providing speed mentoring/training sessions (guided by senior colleagues highly involved in education, mentoring and/or research) for YCPs, as well as a place where motivated YCPs can give their congress speeches/lectures.

\section{Development (2017-2021)}

An exploratory survey was delivered in 2016 within National Boards of the European Society to characterize expertise in $\mathrm{CP}$ and potential career opportunities. Despite receiving few results, the approach served to consolidate a first collaborative initiative. Two pre-meetings were celebrated - in 2017 (Prague, Czech Republic) and 2019 (Stockholm, Sweden), in context of the 13th and 14th EACPT congresses, respectively. Both pre-meetings were successful and established a common basis for YCPs to create various networking opportunities.

A second exploratory survey was delivered in 2019 after the congress to recruit future members for the WG. From 2019 onwards, monthly teleconference meetings were scheduled, where members discuss on active scientific collaborations/projects, as well on open education/training options. Conducting collaborative projects strengthen the formal structuring of the WG, as an asset for individual members who are just starting their career and are willing to take part to international research projects. In this setting, a first project on medical cannabis emerged. The project was launched in December 2019, assessing knowledge and attitudes of healthcare professionals from all over Europe. The results have been valued in the form of a scientific article published in a peer-reviewed journal [10].

\section{Objectives for this year and for the future}

The project on the knowledge and attitudes of health professionals towards recreational and medical cannabis has given place so far to our first research publication as a group [10]. The project is still ongoing, with a currently active questionnaire open to European healthcare professionals who are likely to be involved in the management (prescription, delivery, administration, advice) of patients treated with cannabis-based products, whatever their specialty. It is being distributed through national learned societies and different social media. Our plan is to have the results during 2022 and to achieve our second publication in the near future. Progress will be shared in our next pre-meeting. Our next pre-meeting will be held in Athens in 2022 (before the 15th EACPT congress) and will be a great opportunity for the YCPs to collaborate with other colleagues, fostering the establishment of novel multicentric research groups once more. Our goal is to continue providing collaboration between research centres in CP and, when possible, to promote a hub of contemporary active research centres available for internships for residents, as well as $\mathrm{PhD}$, and post-doc positions in $\mathrm{CP}$. We are highly confident that this will ensure representation in our early-stage careers. In addition, we also hope for more opportunities for social and professional gathering such as a congress dinner and other social activities in Athens 2022. These activities will definitely help us to reinforce networking and planned future collaborations.

In 2022/2023, we plan to contact all the European CP national societies in order to obtain and map out the exact number of CP residents/trainees, as well as early career specialists, per each country. On top of that, we will try to collect all national $\mathrm{CP}$ specialty/residency programmes to compare their structure, organization, requirements, duration and acquired competencies. Our intention is to identify international/regional variations in education programmes/opportunities for young clinical pharmacologists across Europe. We plan to present latter overview in form of a new manuscript, on behalf of our WG, in the near future. We believe that this objective is worthy, since it may eventually lay the basis for tailoring a mutual Pan-European $\mathrm{CP}$ education programme and ensure more equal opportunities for young clinical pharmacologists regardless of their regional affiliation.

One of our upcoming future objectives is to support the launching of an annual program for training grants and fellowships within the EACPT. The aim of these grants 
is to encourage and support the development of professional careers of YCPs, and to stimulate and facilitate their attendance to scientific congresses and internships or rotations. Training grants would provide a great opportunity to acquire clinical competence and research experience and would enable YCPs to contribute to their own clinical and academic development, as well as that from their own departments and countries upon return.

Communication is another cornerstone to be further developed within the framework of the WG. Some basic information about our mission is available on the EACPT website [9]. To promote visibility of our WG, we have an account on LinkedIn (EACPT Young Clinical Pharmacologist Network, available at: https://www.linkedin.com/ groups/13742912/), and we are about to create a Twitter account; both platforms are spaces where we will update on our activities and thus engaging communication and creating visibility. Last but not least, EACPT YCP WG is open for new colleagues to join us with the objective to promote $\mathrm{CP}$ in Europe while creating a vibrant and inclusive $\mathrm{CP}$ community that welcomes equality and celebrates diversity. We are willing to work actively towards helping our senior colleagues in setting standards in CP all around Europe and globe, making our future even more significant.

\section{Closing remarks}

Clinical pharmacology is the medical specialty focusing on safe, rational, effective and economic use of drugs. It is highly important in everyday practice (now more than ever due to COVID-19 pandemic situation), since it has both clinical, regulatory, research and academic ties. Thus, it is of high importance to expand its potential and popularity even more in the following years [5, 11, 12]. Early gathering of motivated YCPs, future leaders in this field, within the same umbrella WG is definitely a way to go and a cornerstone for the brighter future of $\mathrm{CP}$. All things considered, we strongly believe that publishing this paper, with a description of our EACPT YCP WG's structure, chronography, objectives, accomplishments and current/future focus areas, is a great opportunity to notably enhance its visibility, increase the number of our members and mobility/networking options (also between different societies and WGs), as well as to expand our areas of activity even more in the following years. Moreover, by this we can steadily make CP more attractive to young fellows and colleagues, empower its position alongside other medical specialties (e.g. internal medicine, anaesthesiology, surgery, oncology) and once for all wake up a 'sleeping beauty'.
Author contribution AB, CSP, EJ, PA, ALA, RR, MŽ, RNA and JSP contributed substantially to this work. All the authors were involved in data acquisition, writing, and critical revision. $\mathrm{AB}$ contributed to the idea conception, manuscript design and overall team coordination as well. RR and RNA constructed the accompanying figure. All the authors gave the final approval of the version to be published. To deduce, all the authors meet the ICMJE criteria for authorship.

\section{Declarations}

Ethics approval This is a review. Hence, no ethical approval is required.

Competing interests The authors declare no competing interests.

\section{References}

1. Aronson JK (2006) Clinical pharmacology: past, present, and future. Br J Clin Pharmacol 61(6):647-649. https://doi.org/10.1111/j.13652125.2006.02685.x

2. Dollery CT (2006) Clinical pharmacology - the first 75 years and a view of the future. Br J Clin Pharmacol 61(6):650-665. https://doi. org/10.1111/j.1365-2125.2006.02672.x

3. Martin JH, Henry D, Gray J, Day R, Bochner F, Ferro A et al (2016) Achieving the World Health Organization's vision for clinical pharmacology. Br J Clin Pharmacol 81(2):223-227. https://doi.org/10. 1111/bcp.12803

4. Orme M, Sjöqvist F, Birkett D et al (2012) Clinical pharmacology in health care, teaching and research. World Health Organisation/ International Union of Basic and Clinical Pharmacology/Council for International Organizations of Medical Sciences, Geneva

5. Coleman JJ, Samer C, Zeitlinger M, van Agtmael M, Rongen GA, Marquet P et al (2019) The European Association for Clinical Pharmacology and Therapeutics-25 years' young and going strong. Eur J Clin Pharmacol 75(6):743-750. https://doi.org/10.1007/ s00228-019-02690-5

6. European Association of Clinical Pharmacology and Therapeutics (1993). Br J Clin Pharmacol 36(3):183-184

7. European Association for Clinical Pharmacology and Therapeutics. European Association for Clinical Pharmacology and Therapeutic - about EACPT. https://eacpt.org. Accessed 19 Nov 2021

8. Peñataro JS, Fernández J, Walker L, Aydin B, Holm J, Geijteman ECT et al (2015) Clinical pharmacology education in Europe: a time to act. Clin Ther 37(8):e17

9. European Association for Clinical Pharmacology and Therapeutics. European Association for Clinical Pharmacology and Therapeutic - young clinical pharmacologists. https://eacpt.org/working-groups/ young-clinical-pharmacologists/. Accessed 19 Nov 2021

10. Jouanjus E, Sans-Pola C, Mainoli B, Javid FA, Ekheden I; Working Group on medical cannabis projects of the EACPT Young Clinical Pharmacologists (2021) establishing and evaluating a study questionnaire on knowledge and attitudes of healthcare professionals towards recreational and medical cannabis across Europe. Clin Drug Investig 41(8):701-710. https://doi.org/10.1007/ s40261-021-01058-x

11. Francetić I, Likić R (2006) Clinical pharmacology-a sleeping beauty? Br J Clin Pharmacol 62(6):717-718. https://doi.org/10. 1111/j.1365-2125.2006.02754.x

12. Maxwell SR, Webb DJ (2006) Clinical pharmacology-too young to die? Lancet 367(9513):799-800. https://doi.org/10.1016/S01406736(06)68316-5

Publisher's Note Springer Nature remains neutral with regard to jurisdictional claims in published maps and institutional affiliations. 